\title{
"As long as they behave themselves": Heterosexual recuperation in South Africans' talk about homosexuality
}

\section{Abstract}

Several qualitative researchers using discursive methodologies have noted how opposition to homosexuality has not necessarily diminished, despite the general expression of liberal tolerance in many settings. Instead, heterosexist rhetoric has shifted to accommodate political change. Our research builds on this observation within the South African context, using a discursive psychology approach. We examine rhetorical strategies of "heterosexual recuperation": the ways that heterosexual boundaries and the dominance of heterosexuality are maintained by speakers, at the same time as they attempt to avoid being heard as heterosexist. Drawing on data from a qualitative study conducted with heterosexual-identifying Black South Africans (32) from four provinces, we focus on talk that was resourced by a "discourse of tolerance" and characterised by speakers' concern to avoid the attribution of heterosexism. This talk was analysed using thematic analysis, to which discursive psychology techniques were applied. We identified two ways of speaking that relied on this discourse - (1) "As long as they do it in private", and (2) "Flashing their homosexuality" - and show how they ultimately worked to recuperate heterosexuality and marginalise non-normative sexualities. We discuss the implications of these findings in relation to a critical psychology that works to challenge hetero-patriarchal norms.

\section{Introduction}

The measurement of public acceptance of non-normative sexuality through survey and attitude scales has pointed toward higher levels of acceptance in most progressive contexts, where sexual minorities have been afforded formal legal protections (Pew Research Centre, 2013). In

\section{Tracy Morison*}

School of Psychology,

Massey University,

Palmerston North,

New Zealand

t.morison@massey.ac.nz

\section{Amanda Mtshengu \\ Human Sciences \\ Research Council, \\ Pretoria}

\section{Theodorus Sandfort}

Columbia University, New York City, USA

\section{Vasu Reddy}

University of Pretoria, Pretoria

*Corresponding author

\section{Keywords}

attitude, discourse analysis, discursive psychology, heterosexism, homophobia, prejudice, sexuality 
these contexts - usually more secular and affluent - expressions of liberal tolerance are taken by researchers as indicative of positive change in people's attitudes (Clarke, 2005). Yet, several researchers have noted, on the basis of in-depth qualitative investigation, that opposition to homosexuality has not necessarily diminished, despite the general expression of liberal tolerance. Instead opposition has become more refined, with rhetoric shifting somewhat to accommodate political change (Brickell, 2001; Burridge, 2004; Korobov, 2004; Michael, 2013). Even in more secular and affluent contexts where there appears to be a general acceptance of homosexuality, the dominant culture continues to be hetero-centric. As a result, "'public spaces' remain defined as heterosexual spaces, and as sites where heterosexuality is routinely naturalized and homosexuality tightly regulated" (Brickell, 2001: 213).

Qualitative researchers have therefore argued that although it is useful to identify broad trends related to heterosexism, work is needed that facilitates an understanding of the variability and, often, durability of hetero-norms within changing contexts. Indeed, Sigamoney and Epprecht (2013) argue that without a contextual and nuanced understanding, it is difficult to know how to shift prejudices. Accordingly, research is required that allows for an understanding of how people resist or conform to heterosexist norms, including how conformity or resistance becomes situated in the everyday (Korobov, 2004). This includes a need for work that shows how attributions of homophobia/heterosexism might already be taken into account by speakers, so that prejudice may be masked in subtle ways. Our interest in this paper is precisely in the processes that are involved in the production of heterosexism. We focus on strategies of heterosexual recuperation, which, as we explain in more detail further below, refers to the ways that heterosexual boundaries are maintained and the taken-for-granted dominance of heterosexuality is reinforced by speakers, even as they attempt to avoid being heard as heterosexist (Michael, 2013). This is especially evident in talk that draws on a discourse of tolerance, which allows people to avoid being seen as prejudiced, intolerant, or homophobic (Burridge, 2004). Concentrating on such instances is valuable in that it shows the mutability, and indeed durability, of hetero-norms, as well as the ways that people orient to a changing context, in some ways resisting dominant norms while in other ways leaving these intact. Such an investigation can fruitfully shed light on the ways that everyday heterosexism may manifest in ways that are responsive to being heard as prejudiced and that are often less direct and therefore difficult to challenge (Korobov, 2004; Clarke, 2005).

Other than their being a distinct lack of discursive approaches to sexual prejudice in local scholarship, conducting such work in a South African setting is interesting, because the country is in some ways quite different from other contexts where non-normative sexuality is also legally recognised and protected. The legal reform that has occurred 
during the last two decades of the country's democratic transition has generated one of the most progressive constitutions in the world. Yet, rights for sexual minorities, like the rights of many other socially marginalised groups, often remain paper-based, with various contextual barriers, particularly poverty, obstructing their full realisation (Stacey \& Meadow, 2009). There also exists widespread prejudice and discrimination (Nel \& Judge, 2008), with quantitative research indicating that not only are South Africans generally less tolerant toward non-normative sexualities than other liberal democracies, but that the majority (61\%) views homosexuality as unacceptable (Pew Research Centre, 2013). The divide between the official State position and general sentiment could thus be said to be greater than in many other liberal democracies.

In this paper we respond to a gap in the local scholarship on sexual prejudice and adopt a discursive psychological approach to examine talk about homosexuality among a group of heterosexual Black South Africans. (We explain our reason for focusing on this group in particular later in the paper.) The data were drawn from an exploratory qualitative research project in which the aim was to investigate "social attitudes" about non-normative sexualities. We make use of methodological triangulation to grant a perspective on the data that is sometimes not fully attended to by attitudinal research, namely, the situated production of prejudice (Speer \& Potter, 2000). Our interest in this paper is, therefore, not in measuring the levels of prejudice among this particular group of people, or to show that they are or are not more prejudiced than other South Africans. Rather our concern is with how people resist or conform to heterosexist norms in ways that might more subtly perpetuate these norms.

We begin by reviewing some of the key concepts and findings of discursive psychological work on prejudice and sexuality, or heterosexism. Thereafter we provide some background for the project and explicate the specific procedure and key concepts that we used to analyse the data. After presenting our analysis and discussion of our findings, we close by assessing the broader implications of these findings, particularly in relation to the contradictory political context of South Africa.

\section{The situated production of heterosexism or sexual prejudice}

Notions of heterosexism - or "sexual prejudice" (Herek \& McLemore, 2013) - emerged amid growing dissatisfaction with the idea that stigma and prejudice against non-normative sexualities is located within individual attitudes (Clarke, 2005). Feminist and other critical researchers have preferred the term heterosexism to that of homophobia, because it captures the socio-political dimensions of discrimination against sexual minorities, rather than locating sexual prejudice solely within individual pathology (Speer \& Potter, 2000; Herek \& McLemore, 2013). Following on from feminist critiques of homophobia (e.g. Kitzinger, 1987) discursive scholars have theorised the 
notion of heterosexism as "a diverse set of social practices - from the linguistic to the physical ... covert and overt ... in which the homo/hetero binary distinction is at work whereby heterosexuality is privileged" (Plummer, 1992, as cited by Speer \& Potter, 2000: 543). Accordingly, the re/production of heterosexism can be understood as part of the performance of heterosexual identities in which a particular hetero-gendered order is maintained. As Chadwick (2013: 12) argues, heterosexuality is "effected through the use of a variety of arguments and rhetorical positions" that produce its apparent naturalness, and hence invisibility.

Discursive approaches thus emphasise the situated production of heterosexism: the ways that it is produced and negotiated within social interactions. Such work extends and deepens the work that has been done in relation to people's attitudes, beliefs, or opinions about homosexuality and is certainly lacking in the local scholarship on sexual prejudice. With the exception of Chadwick's (2013) study of the rhetorical strategies used by South African women to reproduce and reiterate of normative heterosexuality, this body of work has been produced largely in the "global North".

While the international work does provide some interesting insights, it is imperative that researchers consider how local meanings are implicated in the situated production of heterosexism. As Sigamoney and Epprecht (2013: 84) point out, "Research on sexual minorities often assumes that the meanings of these and other terms are selfevident and fixed in time as general knowledge. The claim that homosexuality is 'unAfrican', for example, has gained notoriety as an articulation of homophobic prejudice throughout the continent. Yet it is an articulation that is heavy with the assumption that homosexuality and 'Africanness' have the same meaning to all speakers, and also that such meanings are translatable across cultural and historical contexts". Following this reasoning, our study utilises a discursive approach that allows us to attend to the subtle, context-sensitive processes involved in local productions of heterosexism (Korobov, 2004), including that of heterosexual recuperation, a concept that we found useful in explaining instances of seemingly tolerant or liberal utterances in the data, and which we explain next.

\section{Heterosexual recuperation}

A number of scholars have explored instances of mundane, subtle, or covert heterosexism in which heterosexist views which are expressed indirectly, often during the course of everyday talk in which heterosexuality is privileged (Clarke, 2005; Peel, 2012). Researchers have examined public talk about sexuality or related topics such as same-sex parenthood (for example, Clarke, 2005) or women's sexuality (for example, Braun, 2000). An exemplary early study by Speer and Potter (2000) utilised a large corpus of British data comprising public talk (such as group discussions, news reportage, 
television documentaries) and one-on-one interviews about gender, sexuality and leisure. Subsequent research has made use of focus group methodologies, including English women (Braun, 2000); undergraduate and/or postgraduate university students (Clarke, 2005); and teenage males (Korobov, 2004, 2005, \& 2006). Others have analysed public debates, for instance parliamentary discussions (Burridge, 2004), and other public talk, such as anti-heterosexism training session data (Peel, 2001) in the United Kingdom.

As Peel (2012: 35) sums up, "One of the interesting, and important, contributions of this body of work is its capacity to capture the subtle, and problematic, aspects of prejudiced talk, despite the positive structural and legal change which has occurred in some countries and jurisdictions over the last decade or so". Our interest in this paper is how speakers accommodate broader discursive shifts within heterosexist talk. Researchers have noted how speakers orient to broader socio-political understandings of sexuality in "the world out there". When talking about sexual minorities, speakers orient toward "what is constructed as normative and accountable in culture" (Speer \& Potter, 2000: 562). Thus, what is "sayable" about sexuality is constrained by the context, for instance in socio-political spaces where tolerance and acceptance of diversity are emphasised, even if only officially, and expressions of intolerance and the attribution of homophobia is undesirable. Recall in South Africa, for instance, how public responses to President Zuma's heterosexist comments lead to an official apology.

Discursive research, such as Speer and Potter's (2000: 562) British study, has shown how prejudiced "speakers use various conversational and interactional resources to portray themselves as reasonable, rational, unprejudiced, [...] and so on". Importantly, Speer and Potter (2000) show how speakers draw on resources that let them express prejudiced views, while at the same time acknowledging and foreclosing potential criticism. Such rhetorical strategies have also been identified by other "Northern" researchers, for example, Clarke's (2005) student focus group discussions about lesbian and gay parenting in the United Kingdom and Korobov's $(2004,2005,2006)$ work with young men in the USA. Both these researchers identified arguments that at first glance appear to display liberal, tolerant, or egalitarian attitudes, but in actual fact mask heterosexism by "inoculating against prejudice". Participants in these studies were able to reinforce "positions of heteronormativity while working to mitigate the threat of being seen as homophobic or sexist" (Korobov, 2004:180).

The strategies described above, we contend, perform the work of "heterosexual recuperation". This term encapsulates "the strategies [used] ... to establish and maintain heterosexual identities without invoking homophobia" (Michael, 2013: 11). Such rhetorical strategies serve to maintain the homo/hetero binary and ultimately reinforce the dominance of heterosexuality, while at the same time "saving face" by avoiding 
being heard as an intolerant or prejudiced person within liberal contexts (Speer \& Potter, 2001; Burridge, 2004). Indeed, researchers have pointed out how speakers may even use liberal language and assumptions to do so and thus indicate "how these are complicit in the reproduction of heteronormativity" (Clarke, 2005: 2).

Researchers have shown how speakers in liberal contexts accommodate contextual change by using liberal language toward conservative ends (Brickell, 2001). Several researchers have noted how a discourse of tolerance plays a central role in "repackaging" old arguments against sexual minority rights, for example in parliamentary debates (Burridge, 2004) or group discussions (Clarke, 2005). This discourse allows speakers to position themselves as "reasonable bearers of liberal tolerance" (Brickell, 2001: 213), while at the same time reinforcing the dominance of heterosexuality. It is drawn on in response to "perceived cultural constraints" (Burridge, 2004: 11), in which prejudice is socially undesirable, and specifically characterised by a concern to avoid the attribution of heterosexism (Burridge, 2004). Thus, as our analysis illustrates, when participants draw on this discourse, it is not to say, that they are necessarily being tolerant, but rather that they wished to appear so. In this way, speakers are able to justify heterosexism, and as we found even violence, similar to Sigamoney and Epprecht's (2013) findings.

An important consideration for work on covert heterosexism, and heterosexual recuperation specifically, has been whether such talk is simply a methodological artefact, resulting due to the researcher's presence (O'hara \& Meyer, 2003). In a British study, Clarke (2005: 8) noted that "the presence of the researcher increases the likelihood of participants attending to concerns about social desirability ... [and] the preponderance of subtle heterosexism ... [in] studies where the researcher was present". However, O'hara and Meyer (2003), in another British study, used a "self-directed focus group" method with no researcher present and report a combination of blatant and subtle heterosexism. This suggests that subtle heterosexism in the research setting could also be fashioned in relation to the broader "world out there": what is socially acceptable when conversing with an unknown person about homosexuality; who might hear or read the research reports.

Like O'hara and Meyer (2003), we also noted a blend of veiled and overt heterosexism across the data set. In South Africa, as in many other liberal democracies, "overt statements about the inferiority of any social group are increasingly frowned upon" (Brickell, 2001: 214). While the situation may certainly be more complex in South Africa, there was evidence in the data of an orientation towards an attribution of heterosexism as unhelpful or undesirable within the context of the interviews. Nevertheless, most of what we know of heterosexual recuperation relates to the Global North, and so our study responds to the need for context-specific work that explores the ways that heterosexism 
is re/produced in the face of contextual changes, such as official recognition of sexual minority rights. Our analysis concentrates on rhetorical strategies that ultimately worked to recuperate heterosexuality and marginalise queer people, despite their seemingly tolerant gloss. Before presenting these findings, we provide some background about the study and the procedures we used in the sections that follow.

\section{About the study}

Our analysis seeks to build on these observations, using a context-sensitive method to explore the variability and complexity views about non-normative sexuality. Quantitative measures of sexual prejudice have identified Black South African's, as a group, as displaying greater sexual prejudice than the general population (Roberts \& Reddy, 2008). Yet, there is some qualitative evidence to contradict the view "that black South Africans are uniformly and incorrigibly homophobic" (Sigamoney \& Epprecht, 2011: 103), which has important implications for advocacy and intervention. Notable in this regard is Sigamoney and Epprecht's (2011: 103) study which showed that "plural codes of conformity and transgression, and acceptance and intolerance, do exist" in particular townships spaces-usually cast as the most intolerant and dangerous settings. Rather than uniform intolerance, the researchers identify instances of what they refer to as "qualified acceptance".

Likewise, in our own study we demonstrate further evidence contesting broad-brushstroke constructions of Black South Africans as uniformly intolerant. We found expressions of both unreserved tolerance and, like Sigamoney and Epprecht (2011) qualified acceptance. In this paper we concentrate specifically on the latter and note that "qualified acceptance" also functions as heterosexual recuperation. Our study therefore provides further local evidence regarding the multifaceted, contradictory, or strategic ways in which Black South Africans actively construct heterosexism in their talk. We discuss the implications that these findings have for opportunities for change at the end of the paper.

\section{Participants}

Participants were recruited through snowballing, as we enlisted participants through social networks and referrals from participants. In order to take part, people were required to identify as Black (or African) and heterosexual, as well as be aged 18 years and older, and living in or near Cape Town, Durban, Johannesburg, and Pretoria (4 research sites). Other than stipulating "race" as an inclusion criterion, we sought to obtain diverse participants, specifically in terms of age, location, gender. Our final group comprised 32 people, with 8 participants per research site. In terms of gender, there was an even split between women and men. The age of participants ranged from 19 to 59 years old, with an average age of 33.5 years. A larger number of the participants 
resided in urban areas (25) than rural areas (7), although many reported having originally come from a rural or peri-urban area. There were also a larger number of students than expected in the sample; half of the participants reported being part-time (8) or full-time (8) students. This meant that the average education level of the group was fairly high: three quarters ( $75 \%$ ) of participants reported that they had completed secondary education, while 21.9 per cent reported attaining secondary education and only 3.1 per cent stating that they had not completed primary education. A greater number $(71.9 \%)$ reported receiving a regular income, than not $(28.1 \%)$. (The major demographics are summarised in Table 1 below and a more detailed description of the participants is added as an Appendix 1.)

Table 1: Summary of participant demographics $(n=32)$

\begin{tabular}{|l|l|l|}
\hline \multirow{3}{*}{ Gender } & Female & 16 \\
\cline { 2 - 3 } & Male & 16 \\
\hline \multirow{5}{*}{ Agcation cohort } & Urban (city and township) & 25 \\
\cline { 2 - 3 } & Rural & 7 \\
\hline \multirow{5}{*}{ Occupation } & $18-28$ & 14 \\
\cline { 2 - 3 } & $29-39$ & 8 \\
\cline { 2 - 3 } & $40-49$ & 7 \\
\cline { 2 - 3 } & $50+$ & 3 \\
\hline & Employed & 25 \\
\cline { 2 - 3 } & Unemployed & 7 \\
\cline { 2 - 3 } & Student (and un/employed) & 16 \\
\hline
\end{tabular}

\section{Interview procedure and dynamics}

Prospective participants were invited to take part in an interview for a study to help "understand more about people's ideas about sexuality and same-sex sexuality in particular". Participants were required to take part in a semi-structured face-to-face interview about sexuality in a language they felt comfortable with. The interviews were conducted in English (15); Zulu (5); Xhosa (4); and Pedi (8) with interviewers fluent in these languages. (Participants could, and did, switch between languages.) $)^{1}$

\footnotetext{
1 Interviews were translated into English where necessary, and back-translated to check the veracity of the translations.
} 
The interview guides contained a number of questions related to the participants' views and beliefs related to sex and sexuality (for example the reasons that people have sex, female and male sexuality, religious views) and non-normative sexualities in particular (for example personal understandings and perspectives, community responses). We were aware that the disclosures might be viewed as embarrassing or personal. Participants were asked to communicate discomfort, and also informed of their right to refuse to answer or to terminate the interview. Similarly, we were cognisant of how disclosures may be shaped by interviewers' identities and personal characteristics, particularly their sexuality, given the nature of the study. Following the concerns raised by the literature regarding researcher presence and social desirability (discussed earlier), interviewers did not to disclose their sexual identity prior to or during the interview. Sexuality cannot be readily inferred - unlike age and gender (from appearance) or expert status (made salient within an academic study) - and so participants did not know whether the interviewers themselves were also heterosexual or not. None asked about this.

We also considered the impact of gender dynamics, due to the sensitivity of the topic, since communication about sexual matters across gender is reported to be difficult in African cultures (Marsten \& King, 2006). We therefore attempted as far as possible to pair researchers with participants by gender where possible. The majority of the interviews were conducted by young Black female and male postgraduate students who could be considered "insiders", like the participants in regard to language and/or cultural background; although their age or educational status could also position them as outsiders. (See the Appendix 1 for a summary of which interviews were conducted by whom and in what language.)

Despite these considerations with regard to researcher identity and interview dynamics, we understood that in qualitative research, the multiple, sometimes contradictory positions held by researchers and participants simultaneously open up some ways of speaking and close others down (Macleod \& Bhatia, 2008). For example, an older woman participant might for example not explain taken for granted cultural or gendered knowledge to a Black female researcher who is younger and unmarried (e.g. cultural taboos), but may explain information she considers to be exclusive to older married persons. In contrast a participant might explain insider knowledge ("what we Zulu people/women believe") to a cultural outsider. When comparing interviews across the data set, we felt that transcripts do not contain vastly significant differences, but rather contained sufficiently rich data to answer the research questions.

\section{Analysis}

The focus of our analysis is on the ways that people pre-emptively avoid or reject "spoiled identities" associated with prejudice (Bamberg, 2004) (for example appearing 
to be racist, sexist, or heterosexist). Such trouble most often motivates a speaker to do repair work through rhetorical strategies to prevent being seen in a negative light; for example, prefacing race talk by saying "Some of my best friends are Indian" to inoculate against accusations of prejudice toward Indian people (Korobov, 2004; Taylor \& Littleton, 2006). (This strategy of credentialing is explained further in the analysis below.) Speakers may also do rhetorical work to explain away or soften his/her claim, for example by specifying that "most (not all) women are bad drivers". (We also explain the use of softening strategies in the analysis.)

Analytically, one is therefore able to explore how speakers reflexively and creatively perform their identities within their talk in relation to the micro-politics of particular localised discursive contexts (like the interview) as well as the broader discursive environment (like South Africa) (Morison \& Macleod, 2013). In the management of prejudice a speaker may therefore consider what is "sayable" in relation to the listener's social identity, as well as in relation to the setting and society at large. Studying such cases, as mentioned, helps to illuminate the complexity of heterosexism, including ways that people orient to a changing context and how everyday heterosexism may manifest in indirect and often insidious ways (Korobov, 2004; Clarke, 2005).

In the analysis that follows we demonstrate how participants' talk orients not only to the immediate context of talk-in-interaction, but also to the broader discursive context (Morison \& Macleod, 2013). We also show how drawing on a discourse of tolerance was interactively useful in that it helped strengthen the rhetorical force of speakers' claims. We identified two rhetorical strategies that relied on this discourse: (1) "As long as they do it in private", and (2) "Flashing their homosexuality". Both these strategies rest upon the construction of an apparently reasonable condition or restriction for in/tolerance and "attempts to justify differential treatment by exceptional or special reasons" (Burridge, 2004: 339). In the first, as we shall show, tolerance is based on keeping certain behaviour private. In the second, tolerance is restricted to only certain "good" homosexual persons. In this way, disapproval is targeted at "something other than a singular, aggregated homosexuality" (Burridge, 2004: 339), such as specific behaviours or persons. The implication is that when this condition is not met, then tolerance falls away and discrimination could be justified.

We discuss each of these strategies in more detail below, showing how they support certain justificatory strategies related to discrimination, and even violence, aimed at gay men and lesbians. We also discuss the rhetorical work that was undertaken to support this talk and repair interactional trouble, using extracts from the data for illustration. The strategies that we present in the analysis serve the discursive function of heterosexual recuperation and were drawn on by the majority of the participants. The extracts that 
appear in the analysis are therefore exemplary of particular rhetorical strategies that appeared across the data set. Each extract includes contextual information regarding the participant's gender and the location of the interview. The transcription conventions that we used are included in Appendix 2. Parts of the extracts that we wish to draw to the reader's attention are marked in bold font.

\section{(1) "As long as they do it in private": Constructing a "reasonable" condition for intolerance}

Participants frequently invoked the notion of privacy in relation to sexuality, for example, describing sex as "a private matter" (Vusumzi ${ }^{2}$ ) or as "very intimate bedroom issues" (Karabo). Drawing on this discursive resource, homosexuality was construed as something that ought to remain unseen. For instance, Gugu (female, urban, Durban) commented: "I think they [the community] accept the [policy?] that homosexuality exists [...] but I think it's like, 'OK, we know you are gay, we don't want to know what you do in the privacy of their own home!'”. Thus, acceptance or tolerance of non-normative sexuality was constructed as tolerable only behind closed doors, in other words, on condition that it is concealed. As the extract below illustrate further, the notion of privacy potentially allowed speakers to avoid being positioned as heterosexist while still justifying discrimination.

\section{Extract 1}

Interviewer: "OK what do you personally think about people engaging in sexual intercourse who are of the same sex" =

Kabelo: = "same sex wow! (.) My emotions towards that, like, for me it's not something that I, uh, I promote. I don't really think it's, it's something that (.) that is good, but possibly, like, for me, it's ... (.) I just disassociate myself with that hey".

Interviewer: "So you di- you disassociate yourself with the (.) with people who are engaging in" =

Kabelo: = "NO, NO, like, the whole activity, like, I don't really like" =

Interviewer: = "disapprove of it?" =

Kabelo: = "Ja, like, I disapprove of it and I don't really like (.) maybe if I meet a homosexual person I won't treat them badly or anything, I just don't wanna know about your sexual life

2 All names in the paper are pseudonyms. Identifying information has been altered where necessary. 
and all of that. The less I know the better. [...] I know people in homosexual relationships, but whether they have sex with each other I don't know. Like I said, uh, their private lives are really I don't really entertain. Like I know them pers- that's why, like, I know them personally, and I know them, like, on a more, like, human being kinda scale so (.) I just, I just, for me they're living their lives they they, they're, uh, (.) doing what they're doing (.) I'm not the one to judge, but you know, it's not something that I entertain".

\section{Extract 2}

Interviewer: "[Marriage rights for sexual minorities] should not be allowed? Government should not allow it?"

Ayanda: "Ja, it should be a private thing [...] We've got people like that here. I don't have problem with them, they go home they do their own things in their own time. I don't mind them. I just take him, take them, as human being[s]. [...] I am not the one to JUDGE them, like (.) they must move here and stay somewhere else. No, as long as they BEHAVE themselves in a right way, I don't have problem with that".

Interviewer: "What is the right way?"

Ayanda: [laughs] [inaudible]

Interviewer: "OK, what don't you want to see?"

Ayanda: "You see to do (.) uh, private things in a street, whatever, in front of kids, whatever. They can do their own things in [a] private house, not in a public in front of children, because children are younger, children who still want to learn from elders."

Interviewer: "So you think that homosexuals whatever they do, they must do it together in private?"

Ayanda: "Yes, somewhere else, not in a public or here amongst us, or here in a community, because really it looks bad, um, when you see a boy here wearing a skirt, walking like this 'HEY, HEY!' [Mimicking 'camp' behaviour] [Laugh] And then we have got young boys here, so young boys they will say, 'That is good. I can do that also."

From the above we can see how sexuality was constructed as part of people's "private lives" (extract 1 ) and involving "private things" (extract 2). In extract 1, there is an explicit conditional - a device common to the management of prejudice talk (Korobov, 2004) - marked by the phrase "I just don't wanna know". Although Kabelo overtly 
states that he would not judge or discriminate against homosexual people, this tolerance is premised upon the condition that sexuality remains hidden, that he does not know about homosexual people's "private lives". Likewise, in extract 2 Ayanda constructs a public/private binary, as well as a them/us distinction in which "us" is discursively aligned with the "public". Tolerance is also dependent on homosexual persons not doing "private things" in public spaces, which Ayanda describes as "behaving themselves". The notion of "behaving" appropriately was a common trope in the data set and usually referred to minimising the visibility of sexuality through gender conformity (as in Ayanda's reference to effeminate clothing and implied "camp" behaviour) and limiting public displays of affection or sexualised behaviour (like kissing or holding hands). In this way, the speakers construct a kind of "live and let live" approach, which is ostensibly tolerant. They position themselves as reasonable, since the condition for tolerance is simply keeping private behaviour private. Sigamoney and Epprecht (2011: 101) report that in the townships they researched, "harsh opinions against homosexuality could be assuaged by the exercise of discretion on the part of same-sex attracted individuals and their maintaining the appearance of heteronormativity" - often through heterosexual marriage and childbearing.

In addition, advocating discrimination was managed in such a way that it also appeared to be consistent with general values of tolerance (Burridge, 2004). This is evident in extract 2. By constructing certain behaviours as inappropriate and unreasonable public displays, homosexual people are positioned as accountable for their openly "extreme" displays of sexuality and, potentially, for any censure that arises as a result. This position is strengthened as the argument shifts to focus onto the 'effects' of homosexuality, rather than homosexuality per se (Burridge, 2004). The speaker mobilises an emotive and common argument about the potential negative effects of children's exposure to homosexuality, especially boys (Clarke, 2001; Clarke \& Kitzinger, 2005; Morison \& Reddy, 2013). Thus, homosexuality is constructed as undesirable in a common-sense way, with no real attempt to justify the reasons for this other than the assumption of non-heterosexuality as a deficit (Clarke, 2005).

A great deal of rhetorical work in both of these extracts supports this positioning. We see how both speakers overtly claim ostensibly non-judgemental positions, invoke the humanity of homosexuals and make use of "credentialing", that is providing evidence of tolerance (Burridge, 2004), for instance, claiming to "personally" (extract 1) know homosexual people. Both speakers initially avoid outright denouncing homosexuality. In extract 1 Kabelo only explicitly states that he disapproves when pressed by the interviewer. Otherwise, we see a reflexive awareness of potential interactive trouble demonstrated by hesitation, frequent pauses, false starts, and self-repair (Korobov, 2004) as well as claims that are moderate and indirect. There is a fair amount of 
rhetorical work that works to repair this in advance, notably his use of the softener "really" and the disclaimer "I'm not one to judge, but" (Burridge, 2004; Korobov, 2004). There is also evidence of repair work in relation to the trouble introduced by the researcher's (unfinished) question. His repair involves constructing intolerance as aimed at homosexuality as an "activity" rather than at "people". This rhetorical strategy, reminiscent of the "love-the-sinner-hate-the-sin" rhetoric of some Christian churches (Mak \& Tsang, 2008), allowed speakers to display tolerance or acceptance of homosexual people while still condemning homosexuality. The presence of such rhetorical work suggests "an orientation towards an attribution of homophobia as unhelpful or undesirable in this context" (Burridge, 2004: 327).

\section{(2) "Flashing their homosexuality": Restricting intolerance to "dangerous queers"}

A second, related rhetorical strategy involved restricting tolerance to "good homosexuals" while condoning discrimination against "dangerous queers" (Burridge, 2004: 330). The distinction was made on the basis of increased visibility as gay or lesbian. For instance, Sibusiso distinguished between those gay men who are "very open" and "show other people that they are homosexual" and an acquaintance of his whom he described as "a typical gentleman ... wearing suits ... you cannot think that he is a homosexual". In this instance, rather than being (primarily) about privacy, the issue is with open contravention of the appropriate hetero-gendered behaviour. This speaks to South African research on the victimisation of [LGBTI] persons in which it was found that "Lesbian, gay and transgender people who are perceived to subvert or undermine patriarchal gender stereotypes, roles and behaviours, are seemingly punished - through discrimination - as a form of social control" (Nel \& Judge, 2008: 26). As we shall see, there were even instances in which such "punishment" was constructed as justifiable on the basis of certain behaviours being constructed as both intentional and "extreme".

The notion of appropriate behaviour, which also emerged within this rhetorical strategy, was thus linked to ideas of appropriate feminine or masculine behaviour, with the "correct" sexual object choice being a member of the "opposite sex". Those who contravene such norms were positioned as flaunting their sexuality, implying that dissent is both wilful and unnecessary. This positioning was resourced by a common stereotype of homosexuals, especially gay men, as promiscuous and hedonistic (Berkowitz, 2007). Dumisani put it like this (notably with some careful rhetorical footwork in the form of hedging): "In terms of lifestyle, (.) Typically, people who practice homosexuality tend to live what one would term an extravagant and a very 'fast' lifestyle. What do I mean by this? I mean being great lovers of groove, being great lovers of material, being show-offers. They live THE social life."

The position of certain homosexual persons as wilful deviants was reinforced by the construction of homosexuality as a "lifestyle", as the quote above shows. This 
construction relies on a liberal discourse that 'emphasises lesbians' and gay men's sameness to heterosexuals and constructs sexuality as a personal choice. Liberalism focuses on the individual and eschews any analysis of power relations between groups" (Clarke, 2005: 3). This in turn supports the construction of the "dangerous queers" whose behaviour is not only extreme, but possibly even wilfully aggravating. Thus, the condition for tolerance was that "homosexuals...must be taught how to behave" (Sibusiso). This rhetoric is illustrated by the following extracts. Both occur at the end of the interviews, after a question about violence against homosexual persons and when the participants were given an opportunity to add anything that they felt had they still wanted to say.

\section{Extract 3}

Tshepo: "I think if you are a gay or lesbian it is up to you to behave. I mean, people overdo this just to show off that "OK I am a gay. I want people to see that am a gay or a lesbian." Some you will not really experience [as] such. You will not be aware of it. [...] So to be a gay it doesn't [mean?] you have to show off the street, to be a lesbian. It is up to you. You know that you are a lesbian; there is no need to wear like (.) also the attire speaks a lot. There are people who know their [status?] somewhere, I think, for people to be terrorised and to be attacked, because they do not behave in the right way."

\section{Extract 4}

Dumisani: [Pause] "There is one; it was also expressed by another homosexual on Metro FM [radio station]. I think that homosexuals must begin to respect themselves. They must limit on wanting to flash their homosexuality as though it's a fashion. And they must limit their extravagant social lifestyle because it goes on to harm them. Why am I saying this? In my understanding, through research it has [been] proven over and over again that there [are] a number of them infected by HIV and AIDS. And I want to attribute such to their extravagant lifestyle, their lavish lifestyle, which involves heavy alcohol abuse and outrageous frequency of having sex, carelessly. [Pause] If they would just limit and begin to respect themselves in that regard, I think we would live in great harmony and we wouldn't have such responses of violence because that kind of lifestyle it makes them to even step on other people's territories. Like if a person wants to, if they're drunk enough, they come on to a guy that is straight [and] he gets angry and feels insulted. And they get a beating-up and that perpetuates violence. So I think they need to begin respecting themselves a lot more and live like normal couples, couples in heterosexual relationship do because they created their own identity, in my understanding, of just MADNESS. In both the practice of sex AND the social extravaganza, it's madness it's on another level. [...] It's preposterous. Really, it's insane!" 
These extracts show the positioning of certain homosexual people as dangerous queers as their behaviour is rendered unnecessary ("no need to") or excessive ("overdo it", "show off", "flashing their homosexuality"). Extreme case formulations strengthen this argument (Speer \& Potter, 2000), especially in extract 4 ("extravagant", "lavish", "social extravaganza"). Again, in both quotes, the behaviour is constructed as intentional, Tshepo says that some people "want" others to know they are homosexual and that "it's up to" a person how to behave. Dumisani speaks of "lifestyles" and maintains that homosexual people "created their own identity".

The construction of "deviant" behaviour as unnecessary and extreme allows prejudice to appear as normative (Speer \& Potter, 2000). Demands for people to minimise the visibility of their sexuality - in order to uphold hetero-patriarchal gender roles and behaviour - are not linked to discriminatory attitudes, but rather constructed as a reasonable request. Therefore, as the extracts above show, "[p]articipants may inoculate themselves from the potential for their remarks to be interpreted as prejudicial or biased, by constructing that view as rationally arrived at" (Speer \& Potter, 2000: 545). For example, in extract 4 Dumisani prefaces his view as one that was originally publically expressed by a homosexual person, thereby discounting the possibility that it is discriminatory. Prefacing the statement in this way gives the argument greater legitimacy: If it is not the product of personal prejudice, "then the speaker must have a more 'rational', stronger reason for making it, and the addressee asked to take it more seriously" (Burridge, 2004: 332). The speaker's rationality is contrasted with the "madness" and "preposterous" or "insane" behaviour of those who are openly gay.

This way of speaking also allowed participants to justify discrimination and, as we see above, potentially even violence, which was construed as an understandable, and possibly even foreseeable, response to provocation. Similarly, Sigamoney and Epprecht (2012: 99) note that their "informants pointed to the imperative to sustain the boundaries separating the norm conformer (the real African) from the norm transgressor (the unAfrican) and in many cases rationalized the use of violence to do so". As a result of this kind of formulation, instead of unjust societal norms and prejudice being seen as problematic, the issue is individualised and, as the extracts above illustrate, sexual and gender non-conforming persons were positioned as responsible for their own suffering and for greater societal conflict. In the quotes above we see that they are portrayed as provoking others by overstepping reasonable bounds ("step on other people's territories") and inviting harm (such as disease and violence) onto themselves. These claims are backed up in extract 4 by reference to objective ("research") as well as experiential evidence. 
In line with this reasoning - and drawing on the stereotype of the promiscuous, hyper-sexed homosexual - another very common theme is alluded to in 4, namely, that heterosexual persons are provoked when homosexuals make unwanted sexual advances. For example, Nokwazi said: "How can I be friends with them, because she will be trying to touch me all the time?" Several participants expressed the sentiment that they would tolerate homosexuality on condition that gay men and lesbians 'stay away' from them or do not make sexual advances. Consider, for instance, the extract from Katlego's interview in which he constructs tolerance of his homosexual friends as conditional.

\section{Extract 5}

Katlego: "Though I hate homosexual activities I don't hate people. I have friends of that kind, but I do not want them close to me when they are drunk. They would try something [with] me, and I think they should stop this behaviour. [...] When they are drunk they become unruly. They [tend?] to think that they can play with everyone forgetting that we are real men and are against their actions, even though they are our friends. You cannot unfriend somebody because they sleep with other men. We're still their friends, but when they are drunk it's better to distance yourself from them, because they think they can play with everyone, even those who are against their actions."

There is much rhetorical work in this extract to repair interactional trouble, allowing Katlego to position himself as heterosexual and appropriately masculine. Credentialing occurs when Katlego mentions that he is friends with gay men. We also see how he distinguishes between homosexual "activities" and "people" and makes use of disclaimers (marked by "but"). He positions himself as tolerant (not someone who "hates people") and even suggests that homosexual persons should be grateful for his forbearance. As this extract shows, failing to adhere to this condition was constructed as disrespectful, intrusive and as exhausting the patience and benevolence of heterosexuals, whom Katlego describes above as "real men". This provided justification for discrimination. Utterances like Katlego's may appear to be more tolerant (than, say, talk about violence), but as other similar work on hetero-masculinity shows, they also work to safeguard traditional masculine values, which rely on the Othering of femininity and sexual minorities (Korobov 2004).

Some male participants made promises of violence or aggression in such cases. Others, like Katlego above, denounced violence, suggesting that one should "distance yourself from them" or that homosexual people must "keep within their own circles" (Nokwazi, Female, rural, Durban). Such responses were depicted as an understandable and reasonable response to provocation, as opposed to, say, an over-reaction and excessive response at what could be taken as a compliment. 
As the extracts show, some participants justified discrimination and societal homophobic violence in particular, by blaming the victim, while at the same time doing rhetorical work to minimise the risk of troubled positioning. It is possible to see that both of the rhetorical strategies that we have discussed cohere around ideas about the in/visibility of homosexuals in general society. Both of these ways of speaking serve to construct the tolerance of homosexuality as conditional. The first is underpinned by notions of privacy and decency. It essentially constructs homosexuality as tolerable only behind closed doors. The second is based on the idea that homosexuals should not "flaunt" their sexuality. It draws on well-known negative stereotypes of homosexuals (as promiscuous, over-sexed, predatory, hedonistic etc.) in order to make this argument and to construct an image of the "dangerous queer" who poses a threat to heterosexual society.

\section{Discussion}

Each of the rhetorical strategies discussed above draws to some degree on a discourse of tolerance and appeals to socially desirable ideals, such as freedom of choice and non-discrimination, that potentially allow for greater sexual diversity and freedom. Though the talk that we analysed outwardly espouses tolerance, and sometimes even overtly condemns heterosexism, it simultaneously upholds particular heteronormative values and assumptions. We have shown how the ideals of privacy and "decent" behaviour were mobilised to justify discrimination in particular circumstances that were discursively rendered as exceptional and "reasonable". They included instances in which homosexual people overtly display their sexuality through their appearance or certain behaviours that were deemed to be a violation of particular norms and a potential threat to particular individuals (especially children) and to society at large. This allowed for the positioning of homosexual people as either "good homosexuals" or "dangerous queers" (Burridge, 2004). Good homosexuals "behave themselves" by minimising the visibility of their non-normative behaviour or appearance, essentially remaining out of sight, and are thus deemed tolerable. Dangerous queers, on the other hand, were those who were described as wilfully and unreasonably violating norms of privacy or decency.

Differentiating between homosexualities also allowed speakers to avoid positioning trouble. They were able to position themselves as not against all homosexuals, and thus having their views heard as simply rooted in prejudice. Rather, their disapproval was cast as focused on certain exceptions and focused on specific unacceptable behaviours. By constructing these behaviours as unnecessary and wilful deviation from the norm, participants could position themselves as reasonable and rational people. Any negative views expressed about homosexuality - including justifications of discriminatory behaviour, and even violence, toward homosexual persons - could be attributed to objective or rational reasons, such as concerns over potential negative consequences of homosexuality (Burridge, 2004). Thus, speakers were inoculated against prejudice. This 
inoculation performs a dual function: It lends rhetorical force to their arguments and also assists in avoiding occupying a spoiled social identity, namely that of homophobe (Korobov, 2004).

Our findings echo those of Sigamoney and Epprecht (2011: 103) who state that "the apparently harsh and dogmatic hostility to homosexuality that people often expressed is also often belied by an element of qualified acceptance, provided that same-sex attracted people use discretion in their public behavior and acknowledge cultural norms of gender, kin, and African identity". The researchers argue that instances of "qualified acceptance" may provide entry points for change. This may well be, but it is important to note Burridge's (2004: 331 ) point that tolerance is often merely a veneer that overlays disapproval "and is not politically innocent".

Unlike talk that explicitly condemns or vilifies homosexuality (which was also present in the data), the talk that we have analysed in this paper works to reinforce heteronormativity in more or less subtle ways, in some instances making heterosexism difficult to detect or challenge. We therefore argue that this talk performs the work of heterosexual recuperation. At the broader discursive level, therefore, the talk works to maintain the boundaries of heterosexuality (Michael, 2013). This is evident in the taken-for-granted manner in which "heterosexuality is both naturalized as universal and privileged as the norm, as a particular form of practice and identity, over other 'non-normative' sexualities" (Richardson \& Munro, 2012: 17). It was clear in our data that non-heterosexuality was assumed to be a deficit (Clarke, 2005). For example, concerns about the effects of exposing children are based on the assumption that children will inevitably turn out to be heterosexual and that this is the best and most appropriate developmental outcome. In contrast, "alternatives" to heterosexuality are viewed as deviance that is to be suppressed (Richardson \& Munro, 2012). Hence, when participants spoke about their aversion to or difficulty witnessing intimacy between same-gendered partners (for example, kissing or hand-holding) the onus was placed on queer people to be responsive to straight people's sensibilities, rather than on heterosexual people to learn to accept sexual diversity. Similarly, the construction of anger or offence as reasonable or understandable responses toward (possible) sexual advances from persons of the same gender suggests that it is offensive to be presumed to be homosexual. This talk therefore upholds the norm of compulsory heterosexuality so that heterosexual practices remain a significant condition for institutional belonging and citizenship (Brickell, 2001; Richardson \& Munro, 2012).

The maintenance of a hierarchical relationship between heterosexuality and homosexuality - in which the former is privileged and the latter subordinate (Brickell, 2001) - is also evident in the differentiation of homo/sexualities. Those who adhere 
most closely to the heteronorm, and thus do not challenge the ideal of compulsory heterosexuality, are positioned as "good". This includes those homosexuals who conform to hetero-gendered norms and "behave themselves" so that they cannot be identified as queer. Essentially, "behaving themselves" translates as behaving as though they were heterosexual. They, for instance, wear gender-appropriate clothing and refrain from publicly displaying sexualised behaviours, especially toward straight people. In contrast, non-conformists and "dangerous queers" are positioned as the unacceptable, abject "Other". This strategy of Othering re/defines the boundaries of hetero/normality through the exclusion of sexual and gender dissidents - subjects that potentially threaten the heteronorm (Butler, 1993). This allows some flexibility by potentially admitting "good homosexuals", while still retaining the superiority of heterosexuality (Brickell, 2001). Queer bodies are provisionally allowed, but within the existing regulatory framework that refuses their sexuality (Michael, 2013). In order to be accepted or at least tolerated in everyday society, gay men and lesbians are required to regulate their behaviour in order to remain invisible. They are not only required to remain marginal in order to coexist with others in society, but also required to take responsibility for their position in society, as well as their victimisation. The cause of rejection, intolerance, and violence was depicted as the failure to appropriately regulate behaviour to accord with the majority/mainstream society, rather than society's negative attitudes and intolerance. This overlooks the material and systemic bases of oppression (Brickell, 2001).

\section{Conclusion}

Despite expressing tolerance or allowing liberal ideals, the talk that we have analysed does not challenge the underlying contempt of homosexuality, but leaves hetero-gendered norms intact, while simultaneously absolving general society of any responsibility for the marginalisation of sexual minorities. Ultimately, these ways of speaking help speakers to recuperate heterosexuality and work in the interests of heteronormativity, relegating queer subjects to the margins of society (Michael, 2013). This finding supports research that has been conducted in other contexts and provides some useful insights into the ways that prejudice toward non-conforming sexualities might be addressed.

Significantly, this work points to the limitation of liberalism as part of a strategy for widespread change. As Clarke (2005) notes, liberal tolerance has often been seen as a desirable development in conventional homophobia research. Yet, examining the ways that liberalism may function in talk problematises this stance. Liberalism, Clarke (2005) argues, is insufficient as a philosophical framework for a critical psychology that would challenge hetero-patriarchal norms. Rather feminist and critical theory may be more fruitful sites, as already shown by the development of the term heterosexism to replace that of homophobia, which itself may cohere with liberal discourse. Instead, mapping out the terrains of heterosexism, as it operates in various contexts, may provide clues for 
possible alternative discourses of homosexuality that do not perpetuate the privileging of heterosexuality. If, as Sigamoney and Epprecht (2011) suggest, instances of qualified acceptance point to possible avenues for combatting prejudice, then intervention strategies will need to actively address this privilege or run the risk of making only superficial changes.

Our study has demonstrated the complexity of heterosexism among a group of heterosexual Black South Africans. We have demonstrated how these participants oriented to a changing context in ways that makes heterosexism more or less covert and insidious (Korobov, 2004; Clarke, 2005). Liberal discourses, such as a discourse of tolerance, allowed speakers to position themselves as "reasonable bearers of liberal tolerance" (Brickell, 2001: 213) while at the same time reinforcing the dominance of heterosexuality. Such talk, Brickell (2001) argues, can therefore still be mobilised toward conservative purposes. As we have shown, such ways of speaking may be used to justify injustice and discrimination. We therefore advocate for further research in other contexts that attends to the situated production of heterosexism in order to assist with understanding the many ways that prejudice manifests and how it may be addressed.

\section{Acknowledgements.}

The authors gratefully acknowledge the researchers who assisted with interviewing: Lesego Ramphele, Jaqueline Tseke, Safiyya Goga, Sihle Dumisa, Ntebo Mahapa, Nadia Sanger, Thandokazi Maseti, and Khunjulwa Mlobeli-Gqabi. Thanks also to Ingrid Lynch for her comments on an earlier draft of this work and Herman Janse Van Vuuren for copy editing the manuscript.

\section{References}

Bamberg, M (2004) Narrative, discourse, and identities, in Meister, J C, Kindt, T, Schernus, W \& Stein, M (eds) (2004) Narratology beyond literary criticism. Berlin \& New York: Walter de Gruyter.

Berkowitz, D (2007) A sociohistorical analysis of gay men's procreative consciousness. Journal of GLBT Family Studies, 3, 157-190.

Braun, V (2000) Heteronormativity in focus group research. Feminism \& Psychology, 10(1), 133-140. 
Brickell, C (2001) Whose "special treatment"? Heterosexism and the problems with liberalism. Sexualities, 4(2), 211-235.

Burridge, J (2004) "I am not homophobic but ...": Disclaiming in discourse resisting repeal of Section 28. Sexualities, 7(3), 327-344.

Butler, J (1993) Bodies that matter: On the discursive limits of "sex". London: Routledge.

Chadwick, R (2013) Queering heterosexuality in the context of sexual violence activism, Gay and Lesbian Issues \& Psychology Review, 9(1), 3-14.

Clarke, V (2001) What about the children? Arguments against lesbian and gay parenting. Women's Studies International Forum, 24(5), 555-570.

Clarke, V (2005) We're all very liberal in our views: Students talk about lesbian and gay parenting. Lesbian \& Gay Psychology Review, 6(1), 2-15.

Clarke, V \& Kitzinger, C (2005) “We're not living on planet lesbian': Constructions of male role models in debates about lesbian families. Sexualities, 8(2), 137-152.

Herek, G M \& McLemore, K M (2013) Sexual prejudice. Annual Review of Psychology, 64, 309-333.

Kitzinger, C (1987) The social construction of lesbianism. London: Sage.

Kitzinger, C (2005) Speaking as a heterosexual: (How) does sexuality matter for talk-ininteraction. Research on Language and Social Interaction, 38(4), 221-265.

Kitzinger, C \& Peel, E (2005) The de-gaying and re-gaying of AIDS: Contested homophobias in lesbian and gay awareness training. Discourse \& Society, 16(2), 173-197.

Korobov, N (2004) Inoculating against prejudice: A discursive approach to homophobia and sexism in adolescent male talk. Psychology of Men and Masculinity, 5(2), 178-189.

Korobov, N (2005) Ironizing masculinity: How adolescent boys negotiate hetero-normative dilemmas in conversational interaction. Journal of Men's Studies, 13(2), 225-246. 
Korobov, N (2006) The management of "nonrelational sexuality" positioning strategies in adolescent male talk about (hetero)sexual attraction. Men and Masculinities, 8(4), 493-517.

Land, V \& Kitzinger, C (2005) Speaking as a Lesbian: Correcting the Heterosexist Presumption. Research on Language and Social Interaction, 38(4), 371-416.

Land, V \& Kitzinger, C (2007) Contesting same-sex marriage in talk-in-interaction. Feminism \& Psychology. 17(2), 173-183.

Mak, H K \& Tsang, J (2008) Separating the "sinner" from the "sin": Religious orientation and prejudiced behavior toward sexual orientation and promiscuous sex. Journal for the Scientific Study of Religion, 47(3), 379-392.

Marston, C \& King, E (2006) Factors that shape young people's sexual behaviour: A systematic review. Lancet, 368(9547),1581-1586.

Michael, J (2013) “Just don't hit on me and I'm fine": Mapping high school wrestlers' relationship to inclusive masculinity and heterosexual recuperation. International Review for the Sociology of Sport, 50(8), 912 - 928.

Macleod C \& Bhatia S (2008) Postcolonialism and psychology, in Willig, C \& StaintonRogers, W (eds) The Sage handbook of qualitative research in psychology. London: Sage Publications.

Morison, T \& Macleod, C (2013) A performative-performance analytical approach: Infusing Butlerian theory into the narrative-discursive method. Qualitative Inquiry, 19(8), 566-577.

Morison, T \& Reddy, V (2013) Familiar claims: Representations of same-gendered families in South African mainstream news media, in Lubbe, C \& Marnell, J (eds) Home affairs: Rethinking lesbian, gay, bisexual \& transgender families in contemporary South Africa. Johannesburg: Fanele Press.

Nel, J A \& Judge, M (2008) Exploring homophobic victimisation in Gauteng, South Africa: Issues, impacts and responses. Acta Criminologica, 21(3), 19-36.

O'hara, L S \& Meyer, M (2003) "I never felt more uncomfortable in my life": University students' discursive constructions of "the lesbian convention". Communication Studies, 54(2), 137-153. 
Peel, E A (2001) Mundane heterosexism: Understanding incidents of the everyday. Women's studies international forum, 24(5), 541-554.

Peel, E (2012) Moving beyond heterosexism? The good, the bad and the indifferent in accounts of others' reactions to important life events. Psychology of Sexualities Review, 3(1), 34-46.

Pew Research Centre (2013) The global divide on homosexuality: Greater acceptance in more secular and affluent countries. Research report.

Accessed August 2014: http://pewglobal.org

Richardson, D \& Munro, S (2012) Sexuality, equality, and diversity. Basingstoke: Palgrave Macmillan.

Roberts, B \& Reddy, V (2008) Pride and prejudice: Public attitudes toward homosexuality. HSRC Review, 6(4), 9-11.

Sigamoney, V \& Epprecht, M (2013) Meanings of homosexuality, same-sex sexuality, and Africanness in two South African townships: An evidence-based ppproach for rethinking same-sex prejudice. African Studies Review, 56(2), 83-107.

Speer, S \& Potter J (2000) The management of heterosexist talk: Conversational resources and prejudiced claims. Discourse \& Society, 11(4), 543-572.

Taylor, S \& Littleton, K (2006) Biographies in talk: a narrative-discursive research approach. Qualitative Sociology Review, 2(1), 22-38. 


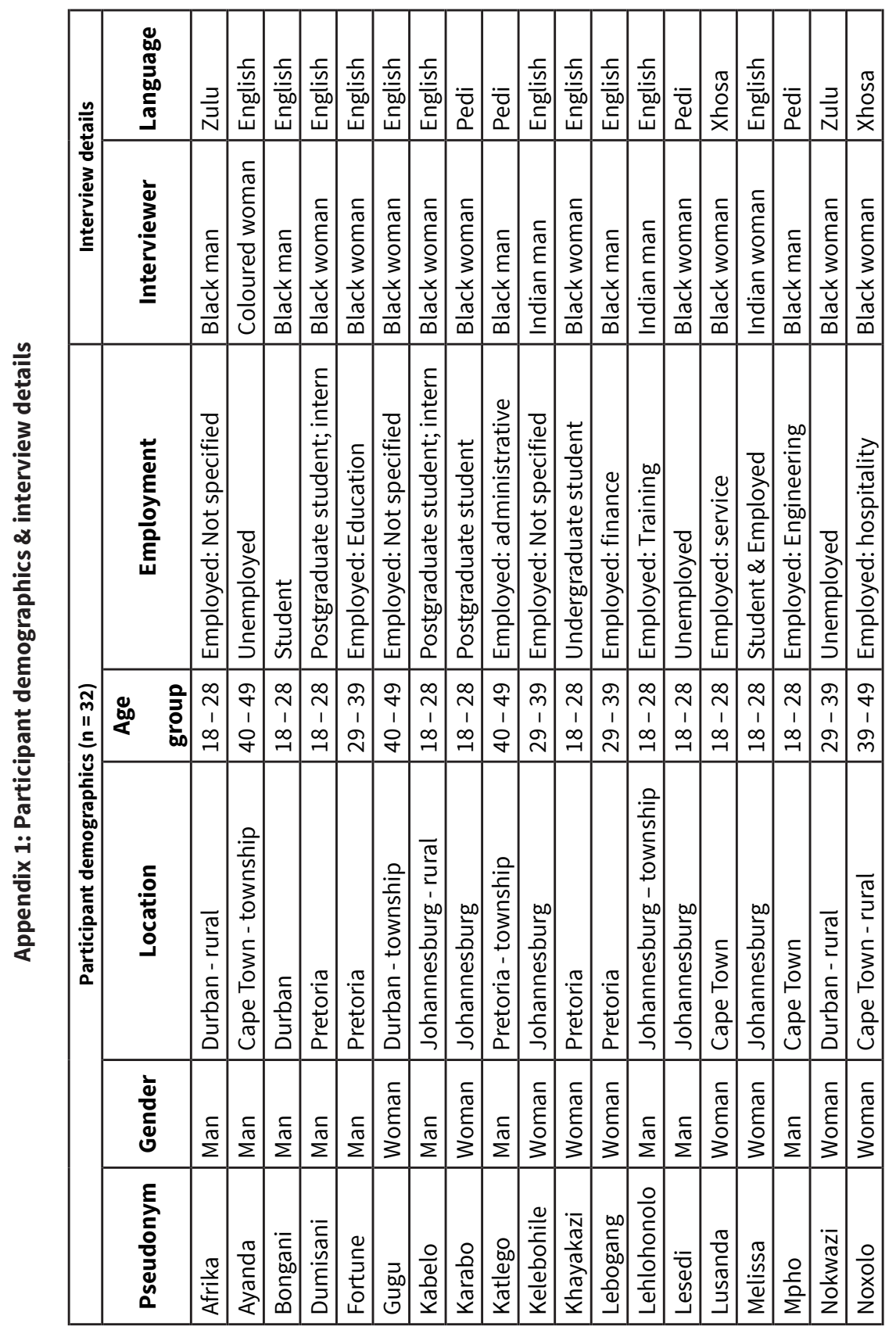

PINS [Psychology in Society] 51.2016|52 


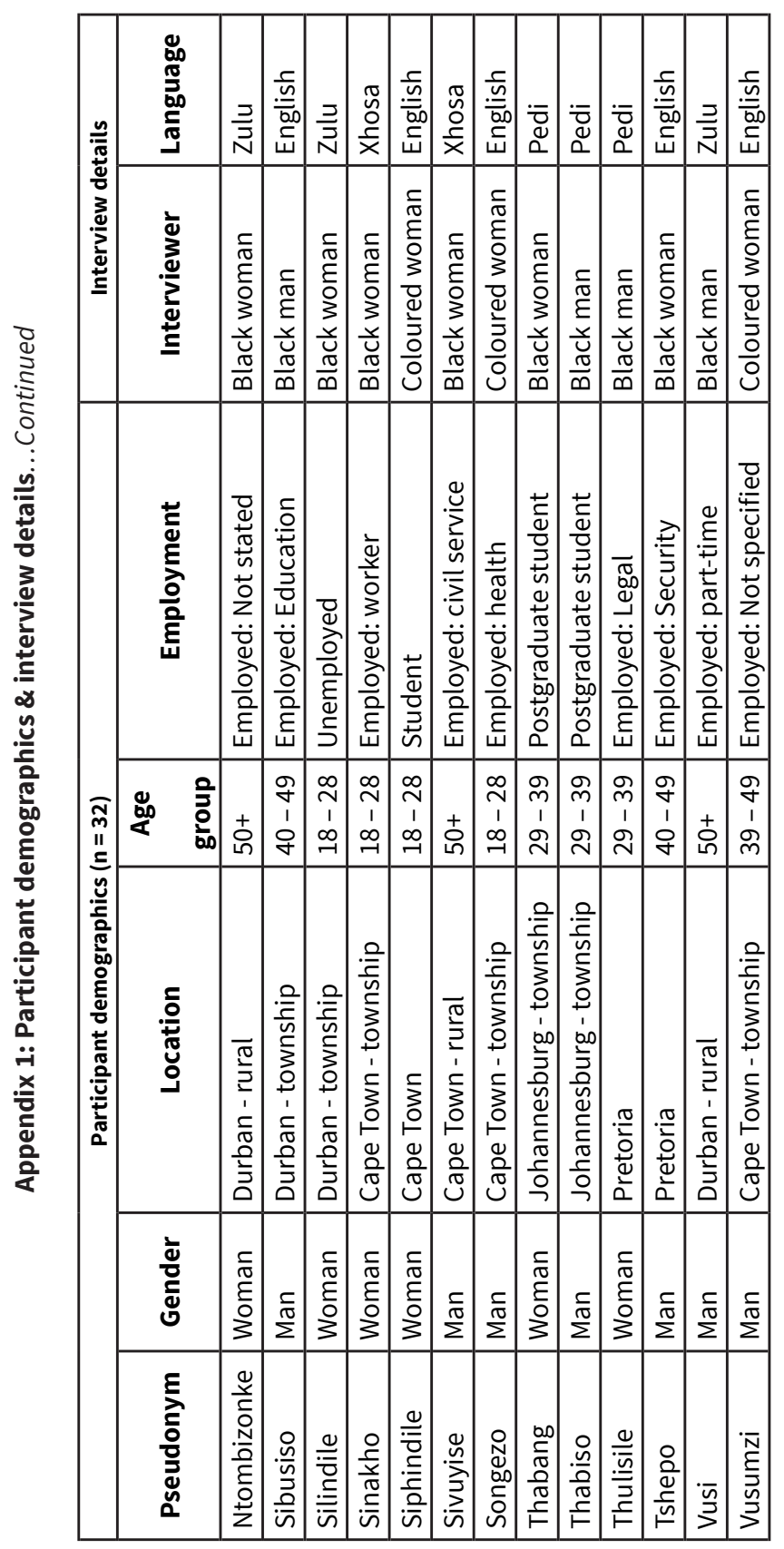

$53 \mid$ PINS [Psychology in Society] 51.2016 
Appendix 2: Transcription conventions used

\begin{tabular}{|c|c|}
\hline$=$ & $\begin{array}{l}\text { At beginning/end of line shows talking over the first speaker/or } \\
\text { interjected a comment }\end{array}$ \\
\hline (.) & $\begin{array}{l}\text { in middle of speaking indicates a speaker's brief space between } \\
\text { spoken words }\end{array}$ \\
\hline [pause] & Indicates a space longer than the brief space of a (.) \\
\hline [laugh] & a short burst of laughter from the speaker \\
\hline [Word?] & $\begin{array}{l}\text { Indicates that the word or phrase in parentheses sounds like } \\
\text { what was heard, but not certain }\end{array}$ \\
\hline$\ldots$ & At end of line means the person trailed off \\
\hline$[x y z]$ & $\begin{array}{l}\text { Indicates editing / clarification (what the speaker probably } \\
\text { meant) as well as additional comments from transcriber ( e.g., } \\
\text { context or intonation) }\end{array}$ \\
\hline$[\ldots]$ & Text omitted because irrelevant or repetitive, to shorten extract \\
\hline CAPITALS & $\begin{array}{l}\text { Capitals mark speech that is obviously louder than surrounding } \\
\text { speech (contrastive emphasis) }\end{array}$ \\
\hline
\end{tabular}

